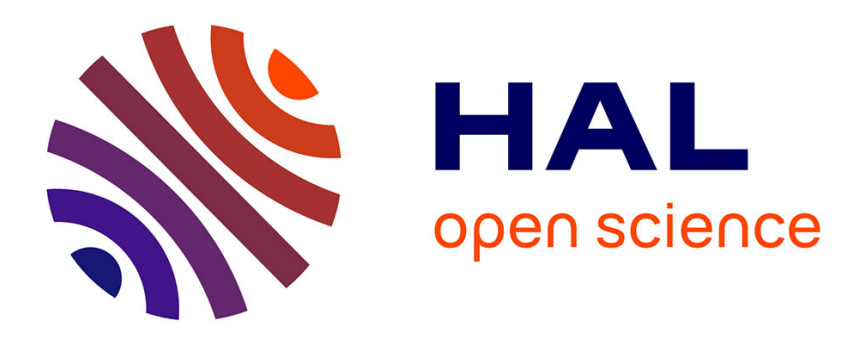

\title{
Stabilization via homogeneous feedback controls Emmanuel Moulay
}

\section{To cite this version:}

Emmanuel Moulay. Stabilization via homogeneous feedback controls. Automatica, 2008, 44 (11), pp.2981-2984. 10.1016/j.automatica.2008.05.003 . hal-03088808

\section{HAL Id: hal-03088808 https://hal.science/hal-03088808}

Submitted on 19 Jan 2021

HAL is a multi-disciplinary open access archive for the deposit and dissemination of scientific research documents, whether they are published or not. The documents may come from teaching and research institutions in France or abroad, or from public or private research centers.
L'archive ouverte pluridisciplinaire HAL, est destinée au dépôt et à la diffusion de documents scientifiques de niveau recherche, publiés ou non, émanant des établissements d'enseignement et de recherche français ou étrangers, des laboratoires publics ou privés. 


\title{
Stabilization via homogeneous feedback controls *
}

\author{
Emmanuel MOULAY ${ }^{\mathrm{a}}$, \\ ${ }^{\mathrm{a}} \operatorname{IRCCyN}($ UMR-CNRS 6597) - Ecole Centrale de Nantes \\ 1 rue de la Noë - B.P. 92101 \\ 44321 Nantes cedex 3 - France
}

\begin{abstract}
In this paper, we provide an explicit homogeneous feedback control with the requirement that a control Lyapunov function exists for an affine control system and satisfies an homogeneous condition. We use a modified version of the Sontag formula to achieve our main goal. Moreover, we prove that the existence of an homogeneous control Lyapunov function for an homogeneous affine system leads to an homogeneous closed-loop system by using the previous feedback control.
\end{abstract}

Key words: Control Lyapunov function; Feedback stabilization; Homogeneous system.

\section{Introduction}

The weighted homogeneity, which has been introduced by Rothschild and Stein in [10], plays an important role in nonlinear control theory. This notion was extensively used:

(1) for the construction of a nilpotent approximating system which is small time local controllable and homogeneous (see [4]),

(2) for homogeneous feedback stabilization in $[5,6]$ when searching an homogeneous closed-loop system,

(3) for finite time stabilization (see [1]).

The stabilization of control systems by homogeneous feedback control has also been developed in [2] by using backstepping technics and in [3] by using homogeneous control Lyapunov function for controllable systems.

The paper deals with finding homogeneous continuous feedback laws by using control Lyapunov functions. We develop a modified version of the Sontag formula in order to obtain an explicit homogeneous feedback control. In a first way, it is interesting to find an homogeneous feedback control even if homogeneous feedback stabilization is not possible. Indeed, homogeneous functions

\footnotetext{
* This work was supported in part by AtlanSTIC (CNRS FR 2819)

Email address: Emmanuel.Moulay@irccyn.ec-nantes.fr (Emmanuel MOULAY).
}

possess very interesting properties which allow to restrict their study to compact manifolds (see [8, Lemma 1]). In a second way, finding an homogeneous feedback control is often the first step when dealing with the research of an homogeneous closed-loop system.

We can also mention that certain homogeneous systems cannot be stabilized by homogeneous feedback laws. This has been first pointed out in [9] and in [11] for affine systems.

The organization of this paper is as follow. Section 2 provides some usefull notations and definitions. In section 3 we develop our main result based on a modified version of the Sontag formula. We give an explicit homogeneous feedback control for affine systems which possess a control Lyapunov function satisfying an homogeneous condition. The closed-loop system is not homogeneous in general. In section 4, we give an explicit homogeneous feedback control for homogeneous affine systems which possess an homogeneous control Lyapunov function. In this case, the closed-loop system is homogeneous.

\section{Notations and definitions}

Throughout this paper, $\mathcal{V}$ will be a non empty neighborhood of the origin in $\mathbb{R}^{n}$. Let us consider the system

$$
\dot{x}=f(x), \quad x \in \mathbb{R}^{n}
$$

where $f: \mathbb{R}^{n} \rightarrow \mathbb{R}^{n}$ is a continuous function. If $V$ : $\mathcal{V} \rightarrow \mathbb{R}^{n}$ is a continuously differentiable function, the 
derivative of $V$ along the solutions of the system (1) is defined by

$$
\dot{V}(x)=\langle\nabla V(x), f(x)\rangle .
$$

As it is customary in control theory, a Lyapunov function $V: \mathcal{V} \rightarrow \mathbb{R}^{n}$ for the system (1) is a continuously differentiable positive definite function such that $\dot{V}$ is negative definite. The Lie derivative of $V: \mathbb{R}^{n} \rightarrow \mathbb{R}$ along $f: \mathbb{R}^{n} \rightarrow \mathbb{R}^{n}$ is defined by:

$$
\begin{aligned}
\mathcal{L}_{f} V: \mathbb{R}^{n} & \rightarrow \mathbb{R} \\
x & \mapsto\langle\nabla V(x), f(x)\rangle
\end{aligned}
$$

A function $V: \mathbb{R}^{n} \rightarrow \mathbb{R}$ is homogeneous of degree $d$ with respect to the weights $\left(r_{1}, \ldots, r_{n}\right) \in \mathbb{R}_{>0}^{n}$ if

$$
V\left(\Delta_{\lambda}(x)\right)=\lambda^{d} V\left(x_{1}, \ldots, x_{n}\right)
$$

for all $\lambda>0$ where $\Delta_{\lambda}(x)=\left(\lambda^{r_{1}} x_{1}, \ldots, \lambda^{r_{n}} x_{n}\right)$. A vector field $f$ is homogeneous of degree $d$ with respect to the weights $\left(r_{1}, \ldots, r_{n}\right) \in \mathbb{R}_{>0}^{n}$ if for all $1 \leq i \leq n$, the $i$-th component $f_{i}$ is a homogeneous function of degree $r_{i}+d$, that is

$$
f_{i}\left(\Delta_{\lambda}(x)\right)=\lambda^{r_{i}+d} f_{i}\left(x_{1}, \ldots, x_{n}\right)
$$

for all $\lambda>0$. The system (1) is said homogeneous of degree $d$ with respect to the weights $\left(r_{1}, \ldots, r_{n}\right) \in \mathbb{R}_{>0}^{n}$ if $f$ is homogeneous of degree $d$ with respect to the weights $\left(r_{1}, \ldots, r_{n}\right) \in \mathbb{R}_{>0}^{n}$.

\section{Stabilization by homogeneous feedback con- trols}

Let $m \in \mathbb{N}^{*}$, and consider the following affine system

$$
\dot{x}=f_{0}(x)+\sum_{i=1}^{m} f_{i}(x) u_{i}, \quad x \in \mathbb{R}^{n}, u \in \mathbb{R}^{m}
$$

where $f_{i} \in C\left(\mathbb{R}^{n}\right)$ for all $0 \leq i \leq m$ and $f_{0}(0)=0$ and the closed-loop system

$$
\dot{x}=f_{0}(x)+\sum_{i=1}^{m} f_{i}(x) u_{i}(x), \quad x \in \mathbb{R}^{n} .
$$

Let us recall the definition of stabilization. The control system (2) is stabilizable (respectively continuously stabilizable) if there exists a non empty neighborhood of the origin $\mathcal{V}$ in $\mathbb{R}^{n}$ and a feedback control law $u \in$ $C^{0}\left(\mathcal{V} \backslash\{0\}, \mathbb{R}^{m}\right)$ (respectively $u \in C^{0}\left(\mathcal{V}, \mathbb{R}^{m}\right)$ ) such that:

(1) $u(0)=0$,

(2) the origin of the system (3) is asymptotically stable.
We now recall some usual definitions. A continuously differentiable positive definite function $V: \mathcal{V} \rightarrow \mathbb{R}_{\geq 0}$ is acontrol Lyapunov function for the system (2) if for all $x \in \mathcal{V} \backslash\{0\}$,

$$
\inf _{u \in \mathbb{R}^{m}}(a(x)+\langle B(x), u\rangle)<0 .
$$

where

$$
\begin{aligned}
a(x) & =\mathcal{L}_{f_{0}} V(x), \\
b_{i}(x) & =\mathcal{L}_{f_{i}} V(x), \quad 1 \leq i \leq m \\
B(x) & =\left(b_{1}(x), \ldots, b_{m}(x)\right)
\end{aligned}
$$

As usual, such a control Lyapunov function satisfies the small control property if for each $\epsilon>0$, there exists $\delta>0$ such that, if $x \in \delta \mathcal{B}^{n}$, then there exists some $u \in \epsilon \mathcal{B}^{m}$ such that

$$
a(x)+\langle B(x), u\rangle<0 .
$$

Remark 1 If $m=1$, the small control property is equivalent to

$$
\limsup _{\|x\|_{n} \rightarrow 0} \frac{a(x)}{|B(x)|} \leq 0 .
$$

The limit may very well be $-\infty$.

We set $b(x)=\|B(x)\|^{2}$.

If we want to use a control Lyapunov function to obtain an homogeneous feedback control, we can use the following modified version of the Sontag feedback control.

Lemma 2 If there exists a continuously differentiable control Lyapunov function $V: \mathcal{V} \rightarrow \mathbb{R}_{\geq 0}$ for the control system (2), then it is stabilizable under the feedback control $u(x)=\left(u_{1}(x), \ldots, u_{m}(x)\right)$ defined by

$$
u_{i}(x)= \begin{cases}-b_{i}(x) \frac{a(x)+\left(|a(x)|^{p}+b(x)^{q}\right)^{\frac{1}{p}}}{b(x)} & \text { if } x \in \mathcal{V} \backslash\{0\} \\ 0 & \text { if } x=0\end{cases}
$$

where $p>1, q>1$ are positive real numbers. If furthermore $V$ satisfies the small control property, then the feedback control (4) is also continuous at the origin.

Proof. Suppose there exists a continuously differentiable control Lyapunov function $V: \mathcal{V} \rightarrow \mathbb{R}_{\geq 0}$. Let

$$
E=\left\{(x, y) \in \mathbb{R}^{2}: x<0 \text { or } y>0\right\}
$$

and $\varphi$ a function defined on $E$ by

$$
\varphi(x, y)=\left\{\begin{array}{ll}
\frac{x+\left(|x|^{p}+|y|^{q}\right)^{\frac{1}{p}}}{y} & \text { if } y \neq 0 \\
0 & \text { if } y=0
\end{array} .\right.
$$


As $\varphi$ is defined on $E$, we have to prove that

$$
\begin{aligned}
\lim _{y \rightarrow 0, x<0} \frac{x+\left(|x|^{p}+|y|^{q}\right)^{\frac{1}{p}}}{y}=0 . & \\
& =\lim _{y \rightarrow 0, x<0} \frac{-x|y|^{q}}{p y|x|^{p}} \\
\lim _{y \rightarrow 0, x<0} \frac{x+\left(|x|^{p}+|y|^{q}\right)^{\frac{1}{p}}}{y} & =\lim _{y \rightarrow 0, x<0} \frac{x+|x|\left(1+\frac{|y|^{q}}{|x|^{p}}\right)^{\frac{1}{p}}}{\lim _{y \rightarrow 0, x<0} \frac{|y|^{q-1} \operatorname{sgn}(y)}{p|x|^{p-1}}=0 .}
\end{aligned}
$$

$\varphi$ is continuous on $E$. The fact that $V$ is a control Lyapunov function implies that $(a(x), b(x)) \in E$ for all $x \in \mathcal{V} \backslash\{0\}$. Thus

$$
u(x)=\left(u_{1}(x), \ldots, u_{m}(x)\right)
$$

defined by

$$
u_{i}(x)=-b_{i}(x) \varphi(a(x), b(x))
$$

is continuous on $\mathcal{V} \backslash\{0\}$ and we obtain for all $x \in \mathcal{V} \backslash\{0\}$,

$$
\begin{aligned}
\left\langle\nabla V(x), f_{0}(x)+\sum_{i=1}^{m} f_{i}(x) u_{i}(x)\right\rangle & =-\left(|a(x)|^{p}+b(x)^{q}\right)^{\frac{1}{p}} \\
& <0 .
\end{aligned}
$$

$V$ is a Lyapunov function for the closed-loop system (3)(4), and by using the Lyapunov theorem we know that the origin of the closed loop system (3)-(4) is asymptotically stable.

The proof concerning the stabilization under the small control property is similar to the one given in $[12$, Theorem 1].

A less general version of this lemma was proved in $[7$, Lemma 19]. The Sontag control corresponds to the case $(p, q)=(2,2)($ see $[12])$.

We now give a result concerning single input systems.

Theorem 3 Consider the single input system (2) with $m=1$. If there exists:

(1) a continuously differentiable control Lyapunov function $V: \mathcal{V} \rightarrow \mathbb{R}_{>0}$ for the control system (2) such that $a(x)$ is homogeneous of degree $d_{0}$ and $B(x)$ is homogeneous of degree $d_{1}$ with respect to the weights $\left(r_{1}, \ldots, r_{n}\right) \in \mathbb{R}_{>0}^{n}$,

(2) $p>1, q>1$ positive reals such that $p=\frac{2 q d_{1}}{d_{0}}$, then the feedback control

$$
u(x)= \begin{cases}-\frac{a(x)+\left(|a(x)|^{p}+b(x)^{q}\right)^{\frac{1}{p}}}{B(x)} & \text { if } x \in \mathcal{V} \backslash\{0\} \\ 0 & \text { if } x=0\end{cases}
$$

stabilizes the system (2) and is homogeneous of degree $d_{0}-d_{1}$ with respect to the weights $\left(r_{1}, \ldots, r_{n}\right)$. If furthermore $V$ satisfies the small control property, then the feedback control (5) continuously stabilizes the system (2).

Proof. The fact that $u(x)$ stabilizes the system $(2)$ is given by Lemma 2 .

As $a(x)$ is homogeneous of degree $d_{0}$ and $B(x)$ is homogeneous of degree $d_{1}$ with respect to the weights $\left(r_{1}, \ldots, r_{n}\right) \in \mathbb{R}_{>0}^{n}$, we have:

$$
\begin{aligned}
u\left(\Delta_{\lambda}(x)\right) & =-\frac{\lambda^{d_{0}} a(x)+\left(\lambda^{p d_{0}}|a(x)|^{p}+\lambda^{2 d_{1} q} b(x)^{q}\right)^{\frac{1}{p}}}{\lambda^{d_{1}} B(x)} \\
& =-\frac{\lambda^{d_{0}} a(x)+\left(\lambda^{p d_{0}}\left(|a(x)|^{p}+b(x)^{q}\right)\right)^{\frac{1}{p}}}{\lambda^{d_{1}} B(x)} \\
& =-\frac{\lambda^{d_{0}}\left(a(x)+\left(|a(x)|^{p}+b(x)^{q}\right)^{\frac{1}{p}}\right)}{\lambda^{d_{1}} B(x)} \\
& =\lambda^{d_{0}-d_{1}} u(x) .
\end{aligned}
$$

Let us give an example

Example 4 Consider the system

$$
\left\{\begin{array}{l}
\dot{x}_{1}=-x_{1}^{3}+x_{2} \\
\dot{x}_{2}=-x_{1}+u
\end{array}\right.
$$

and the control Lyapunov function

$$
V(x)=\frac{x_{1}^{2}+x_{2}^{2}}{2} .
$$

$a(x)=-x_{1}^{4}$ is homogeneous of degree 4 and $B(x)=x_{2}$ is homogeneous of degree 1 with respect to the weights $(1,1)$. As

$$
\frac{a(x)}{|B(x)|}=\frac{-x_{1}^{4}}{\left|x_{2}\right|} \leq 0
$$

we deduce from Remark 1 that $V$ satisfies the small control property. Theorem (3) with $4 p=2 q$, for instance $(p, q)=\left(\frac{3}{2}, 3\right)$, implies that the system is continuously stabilizable by the feedback control

$$
u(x)=-\frac{-x_{1}^{4}+\left(x_{1}^{6}+x_{2}^{6}\right)^{\frac{2}{3}}}{x_{2}}
$$

which is homogeneous of degree 3 . 
We now address a result for multi-input systems whose proof can be easily deduced from the previous one.

Theorem 5 Consider the system (2). If there exists:

(1) a continuously differentiable control Lyapunov function $V: \mathcal{V} \rightarrow \mathbb{R}_{\geq 0}$ for the control system (2) such that $a(x)$ is homogeneous of degree $d_{0}$ and $b_{i}(x)$ are homogeneous of degree $d_{1}$ with respect to the weights $\left(r_{1}, \ldots, r_{n}\right) \in \mathbb{R}_{>0}^{n}$ for all $1 \leq i \leq m$,

(2) $p>1, q>1$ positive reals such that $p=\frac{2 q d_{1}}{d_{0}}$,

then the feedback control (4) stabilizes the system (2) and its components $u_{i}(x)$ are homogeneous of degree $d_{0}-d_{1}$ with respect to the weights $\left(r_{1}, \ldots, r_{n}\right)$ for all $1 \leq i \leq m$. If furthermore $V$ satisfies the small control property, then the feedback control (4) continuously stabilizes the system (2).

\section{Homogeneous feedback stabilization}

By homogeneous stabilization, we mean that there exists an explicit feedback control such that the closedloop system is homogeneous. This is the purpose of this section. The following lemma can be found in [8].

Lemma 6 If $V$ is homogeneous of degree $d_{1}$ with respect to the weights $\left(r_{1}, \ldots, r_{n}\right) \in \mathbb{R}_{>0}^{n}$ and if $f$ is homogeneous of degree $d_{2}$ with respect to the weights $\left(r_{1}, \ldots, r_{n}\right) \in$ $\mathbb{R}_{>0}^{n}$, then $\mathcal{L}_{f} V$ is homogeneous of degree $d_{1}+d_{2}$ with respect to the weights $\left(r_{1}, \ldots, r_{n}\right) \in \mathbb{R}_{>0}^{n}$.

We can now give a theorem for homogeneous feedback stabilization using an homogeneous control Lyapunov function. Nevertheless, it is more restrictive than Theorem 3.

Theorem 7 Consider the single input system (2) with $m=1$ where $f_{0}(x)$ is homogeneous of degree $d_{0}$ and $f_{1}(x)$ homogeneous of degree $d_{1}$ with respect to the weights $\left(r_{1}, \ldots, r_{n}\right) \in \mathbb{R}_{>0}^{n}$. If there exists a continuously differentiable control Lyapunov function $V: \mathcal{V} \rightarrow \mathbb{R}_{>0}$ for the control system (2) homogeneous of degree $d$ with respect to the weights $\left(r_{1}, \ldots, r_{n}\right) \in \mathbb{R}_{>0}^{n}$, then the feedback control (5) where

$$
p=\frac{2 q\left(d+d_{1}\right)}{d+d_{0}}, p>1, q>1,
$$

is homogeneous of degree $d_{0}-d_{1}$ and the closed-loop system (3)-(5) is homogeneous of degree $d_{0}$ with respect to the weights $\left(r_{1}, \ldots, r_{n}\right)$. If furthermore $V$ satisfies the small control property, then the feedback control (5) continuously stabilizes the system (2).

Proof. As $V(x)$ is homogeneous of degree $d, f_{0}(x)$ is homogeneous of degree $d_{0}$ and $f_{1}(x)$ is homogeneous of degree $d_{1}$ with respect to the weights $\left(r_{1}, \ldots, r_{n}\right) \in \mathbb{R}_{>0}^{n}$, Lemma 6 implies that $a(x)$ is homogeneous of degree $d+$ $d_{0}$ and $B(x)$ is homogeneous of degree $d+d_{1}$. Theorem 3 implies that the feedback control (5) is homogeneous of degree $d_{0}-d_{1}$.

The closed loop system satisfies

$$
\begin{aligned}
\left(f_{0}+f_{1} u\right)\left(\Delta_{\lambda}(x)\right) & =\lambda^{d_{0}} f_{0}(x)+\lambda^{d_{1}} f_{1}(x) \lambda^{d_{0}-d_{1}} u(x) \\
& =\lambda^{d_{0}}\left(f_{0}+f_{1} u\right)(x) .
\end{aligned}
$$

Example 8 Consider the system

$$
\left\{\begin{array}{l}
\dot{x}_{1}=x_{1}-x_{2} \\
\dot{x}_{2}=x_{1}+x_{2} u
\end{array}\right.
$$

and the control Lyapunov function

$$
V(x)=\frac{x_{1}^{2}+x_{2}^{2}}{2}
$$

homogeneous of degree 2 with respect to the weights $(1,1)$. $f_{0}(x)=\left(\begin{array}{c}x_{1}-x_{2} \\ x_{1}\end{array}\right)$ and $f_{1}(x)=\left(\begin{array}{c}0 \\ x_{2}\end{array}\right)$ are homogeneous of degree 0 with respect to the weights $(1,1)$. Theorem (7) with $p=2 q$, for instance $(p, q)=(4,2)$, implies that the system is stabilizable by the feedback control

$$
u(x)=-\frac{x_{1}^{2}+\left(x_{1}^{8}+x_{2}^{8}\right)^{\frac{1}{4}}}{x_{2}^{2}}
$$

which is homogeneous of degree 0 with respect to the weights $(1,1)$. Moreover, the closed-loop system is then homogeneous of degree 0 with respect to the weights $(1,1)$.

As Theorem 5 generalized Theorem 3 to multi-input systems, it is also possible to generalize Theorem 7 to multiinput systems.

\section{Concluding remarks}

The paper deals with the design of an homogeneous feedback control based on control Lyapunov functions satisfying an homogeneous condition. The control law is derived from the well known Sontag control. When the affine control system and the control Lyapunov functions are homogeneous, the closed-loop system is also homogeneous.

\section{Acknowledgements}

The author is extremely grateful to Sergej Celikovsky for the many discussions he has had about the notion of homogeneity. 


\section{References}

[1] S. P. Bhat and D. S. Bernstein. Geometric homogeneity with applications to finite-time stability. Math. Control Signals Systems, 17:101-127, 2005.

[2] J. M. Coron and L. Praly. Adding an integrator for the stabilization problem. Systems Control Lett., 17:89-104, 1991.

[3] L. Grüne. Homogeneous state feedback stabilization of homogeneous systems. SIAM J. Control Optim., 38(4):12881314, 2000.

[4] H. Hermes. Nilpotent and high-order approximations of vector field systems. SIAM Rev., 33(2):238-264, 1991.

[5] M. Kawski. Homogeneous stabilizing feedback laws. Control Theory Adv. Tech., 6(4):497-516, 1990.

[6] M. Kawski. Homogeneous feedback stabilization, in "New Trends in Systems Theory", volume 7. Progress in Systems and Control Theory, Birkhuser, 1991.

[7] E. Moulay and W. Perruquetti. Finite time stability and stabilization of a class of continuous systems. J. Math. Anal. Appl., 323(2):1430-1443, 2006.

[8] L. Rosier. Homogeneous Lyapunov function for homogeneous continuous vector field. Systems Control Lett., 19:467-473, 1992.

[9] L. Rosier. Etude de Quelques Problèmes de Stabilisation. PhD thesis, Ecole Normale Supérieure de Cachan, 1993.

[10] L. P. Rothschild and E. M. Stein. Hypoelliptic differential operators and niipotent groups. Acta Math., 137:247-320, 1976.

[11] R. Sepulchre and D. Aeyels. Stabilizability does not imply homogeneous stabilizability for controllable systems. SIAM J. Control Optim., 34(5):1798-1813, 1996.

[12] E. Sontag. A universal construction of Arststein's theorem on nonlinear stabilization. Systems Control Lett., 13:117-123, 1989. 